\title{
Psychometric Properties of Early Maladaptive Schemas Questionnaire Short Form-75 Items (YSQ-SF)
}

\begin{abstract}
Background: Early maladaptive schemas are the most important causes for psychiatric disorders. Therefore, this study aimed at validating maladaptive schemas questionnaire short form (YSQ-SF-75) in clinical and normal groups.

Method: This current stud was a cross-sectional and instrument validating research. In this research, 431 subjects were who had referred to psychiatric and psychology centers in Tehran (men: clinical: $33 / 8 \%$; women: clinical: $66 / 2 \%$; normal group: $42 / 7 \%$ ) were selected and assigned to clinical group $(\mathrm{n}=204 ; 47.3 \%)$ and normal (n-227; 52/7\%) group without diagnosis. Demographic questionnaire and Young Schemas Questionnaire Short Form (YSQ-SF-75) were used to validate the questionnaire. To examine validity of the construct, factor analysis-based correlation method was used and internal consistency method was applied to examine reliability. Data were analyzed using SPSS-22 Software.

Results: The Cronbach's alpha results obtained from internal reliability of early maladaptive schemas questionnaire indicated the range of 0/76-0/90 for this coefficient and 0/96 for the whole scale. Moreover, correlations of items set obtained above $0 / 4$ approving that majority of subscales (except for SI, DS, DI, and SS) have satisfactory internal consistency.

Conclusion: the research results showed that this questionnaire is a proper and valid tool to for early maladaptive schemas assessment in normal and clinical adults.
\end{abstract}

Keywords: Maladaptive schema, Psychometric properties, personality Disorders.

\footnotetext{
Affiliations

1- Assistant Professor, PhD, Department of Mental health, School of Behavioral Sciences and Mental Health (Tehran Institute of Psychiatry), Iran University of Medical Sciences (IUMS). Tehran, Iran.

2- PhD Candidate of Clinical Psycology, School of Behavioral Sciences and Mental Health (Tehran Institute of Psychiatry), Iran University of Medical Sciences (IUMS). Tehran, Iran.

3- MA of Clinical Psychology, Tonekabon Branch, Islamic Azad University, Tonekabon, Iran.

4- MA of Clinical Psychology, Tonekabon Branch, Islamic Azad University, Tonekabon, Iran.

5- PhD Candidate of Clinical Psycology. Aja University of Medical sciences. Tehran, Iran.

6- MA of Clinical Psychology, Roudehen Branch, Islamic Azad University, Roudehen, Iran.

7- MA of Clinical Psychology, Tonekabon Branch, Islamic Azad University, Tonekabon, Iran.

Corresponding Author: rdavoodi636@gmail.com
}

Article Info

Authors:

Ghahari $\mathrm{SH}^{1} \odot$

Viesy $\mathrm{F}^{2} \odot$

Kavand $\mathrm{H}^{3}$ 。

Yekefallah $\mathrm{M}^{4} \bullet$

Zandifar $\mathrm{H}^{5} \odot$

Farrokhi $\mathrm{N}^{6 \odot}$

Davoodi $\mathrm{R}^{7^{*} \odot}$

Received: 2020/6/17

Accepted: 2020/8/7

E-Published: 2020/9/5

\section{Citation Subjects:}

Psychological Tests, Mental Disorders, Quantitative Psychology 
فصلنامه يرستار و پزشك در رزم / تابستان 99 / سال هشتم

\section{ويزگى هاى روانسنجى يرسشنامه طرحوارههاى ناسازگًار اوليه فرم كوتاه - VD سوالى (YSQ-SF)}

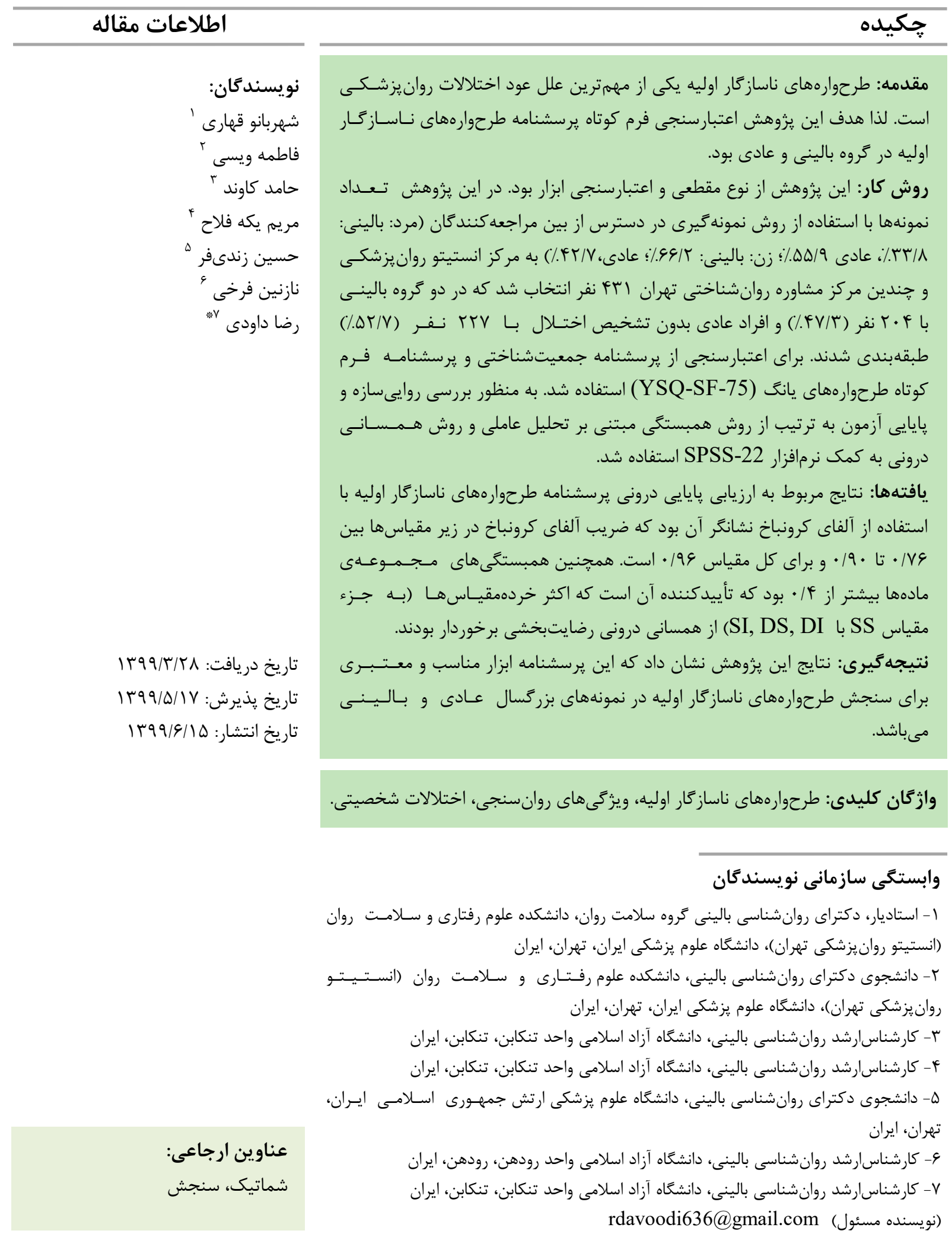


طرحوارههاى سازشنايافته اوليه با ابعاد شخصيتى رابطه دارند و

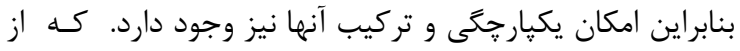

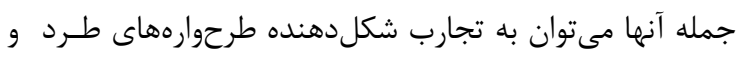

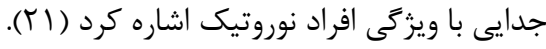

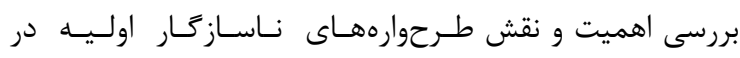

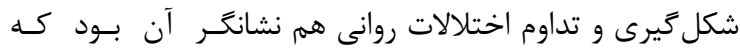

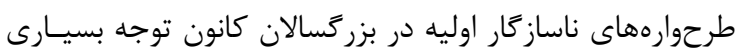

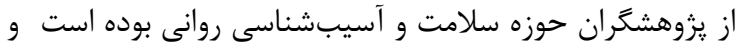

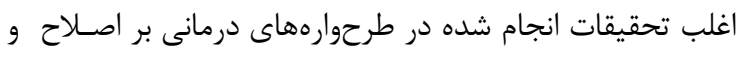

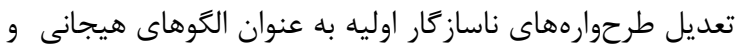

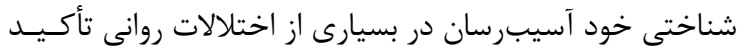

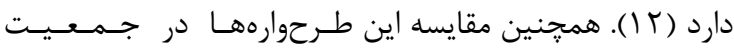

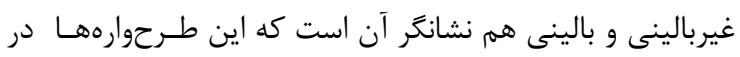

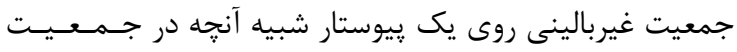

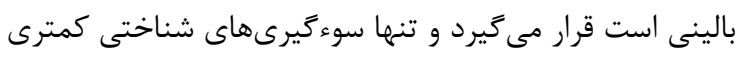

در نمونه هاى غيربالينى ايجاد مى كنند (• (1).

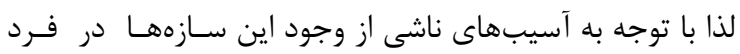

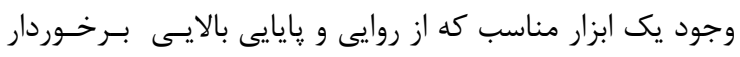

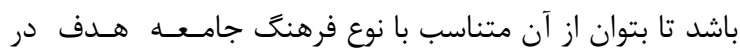
تشخيص بالينى استفاده نمود لازم و ضرورى است. كه از جملـه انه

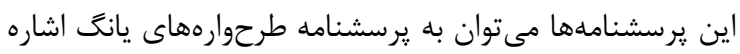

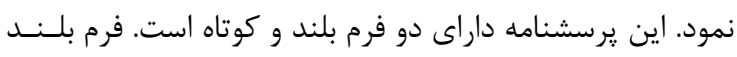

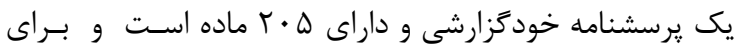

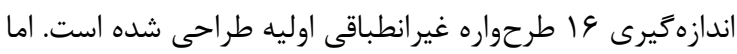
فرم كوتاهتر اين برسشنامه (YSQ-SF-75) به به دليل داشتـن

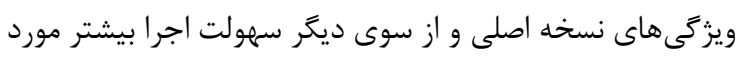

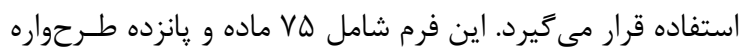

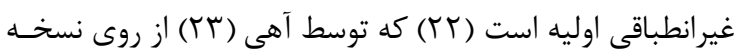

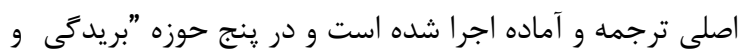

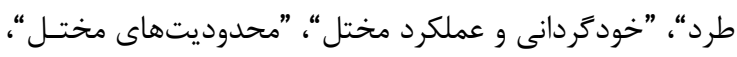

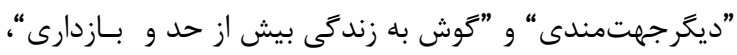

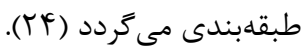

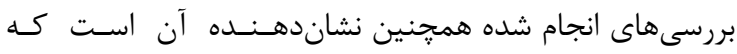

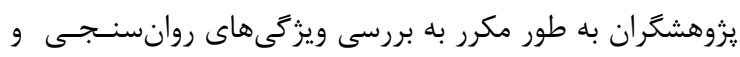

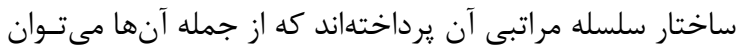

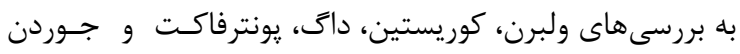

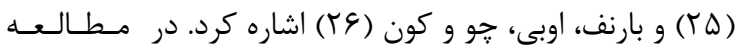

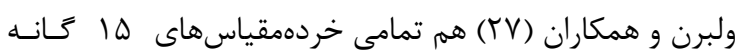

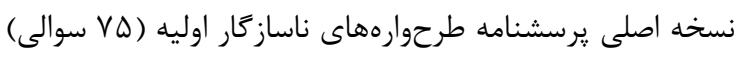

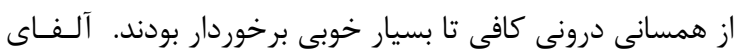

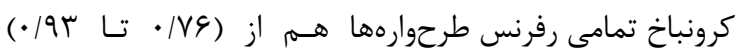

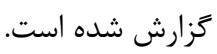

5- Schema Questionnaire- Short Form-75
مقدمه

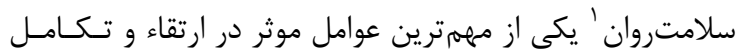

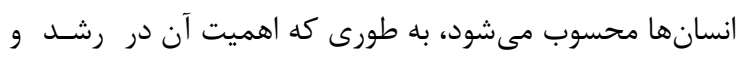

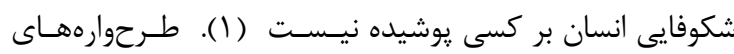

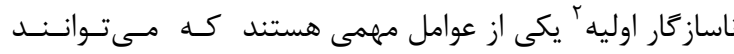

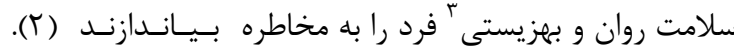

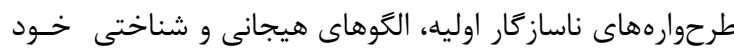

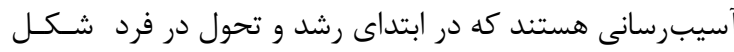

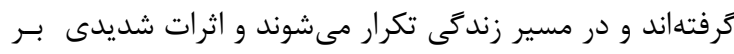

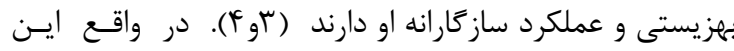

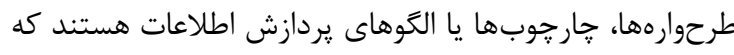

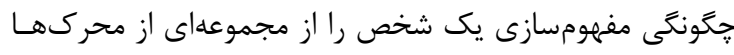

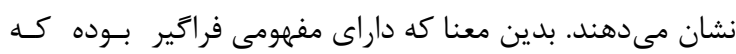

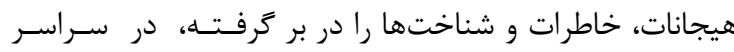
زندگى فرد كسترش مىيابند و استنباط فرد از تجربيات روزانه

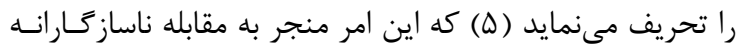

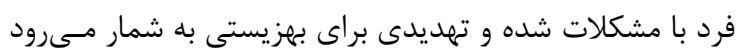

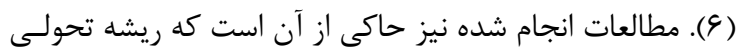

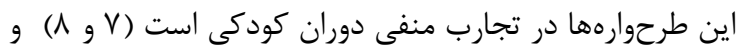

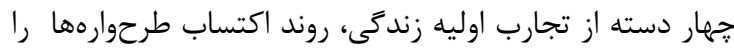

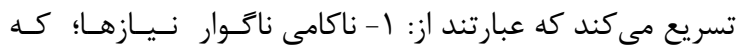

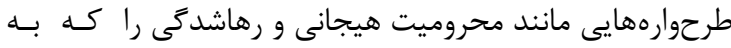

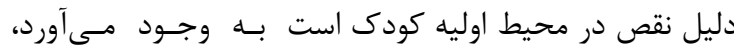

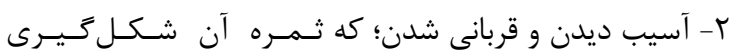

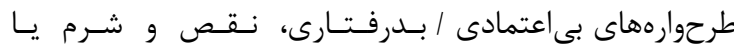

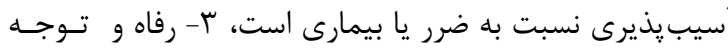

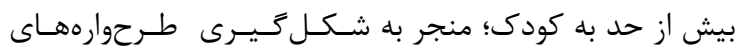

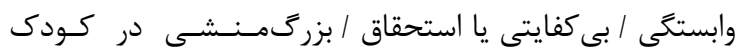

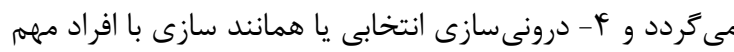

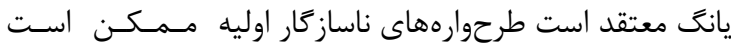

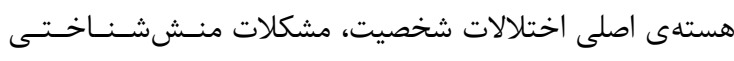

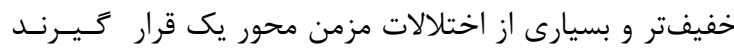

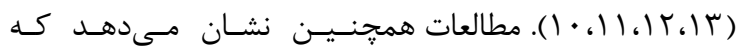

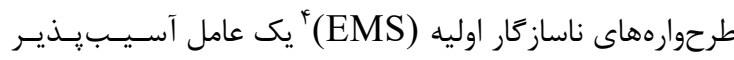

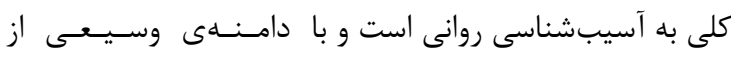

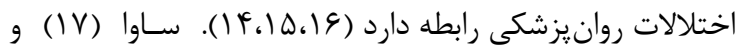

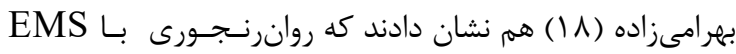

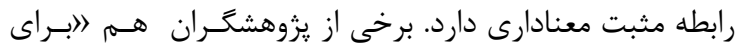

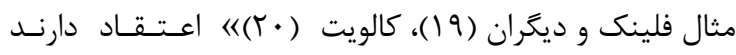

1- Mental Health

2- Early maladaptive schemas

3-well-being

4- Early Maladaptive Schemas 


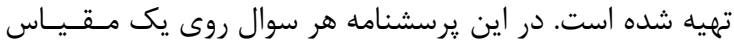

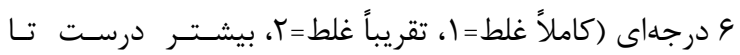

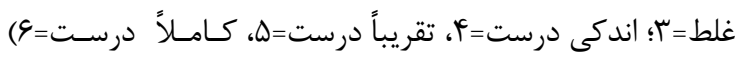

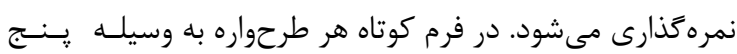

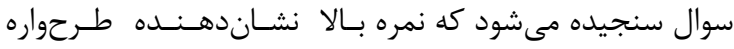

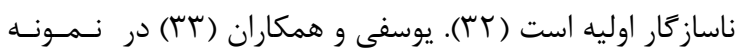

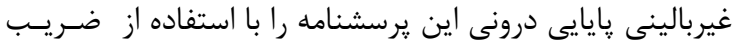

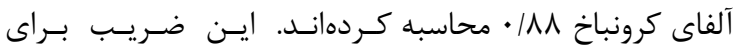

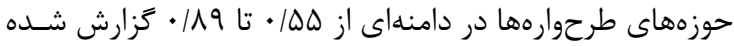

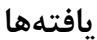

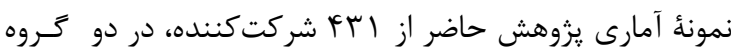

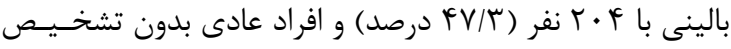

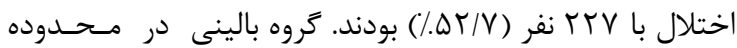

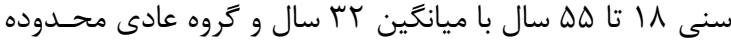

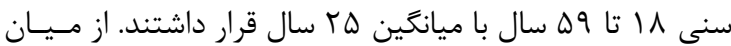

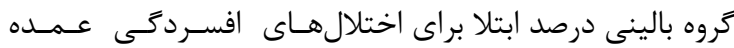

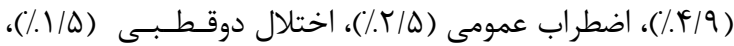

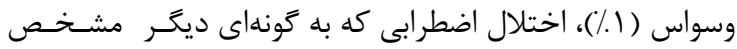

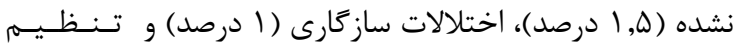

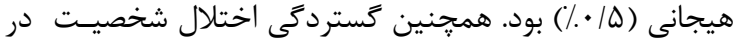

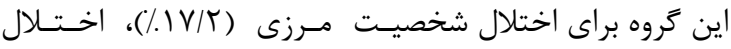

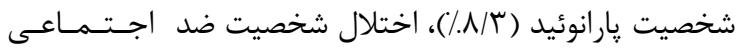

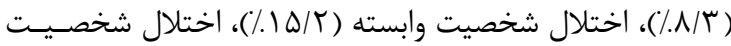

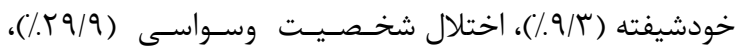

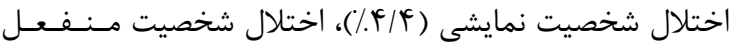

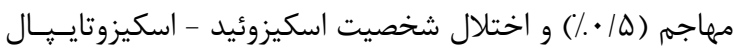

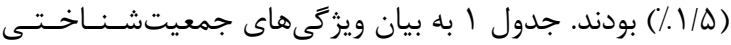
نمونه مورد بر بوسى مى يردازد.

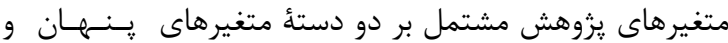

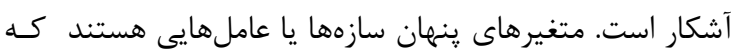

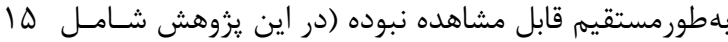

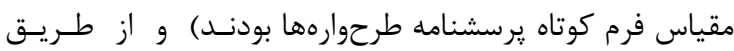

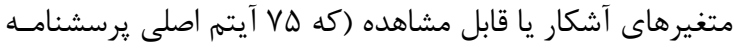

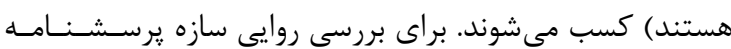

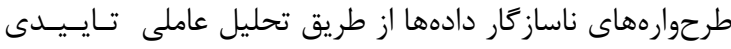

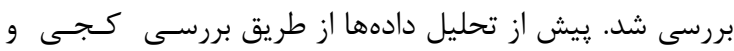

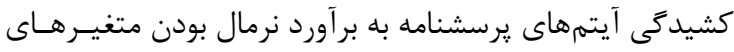

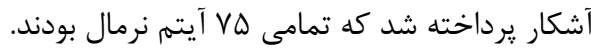

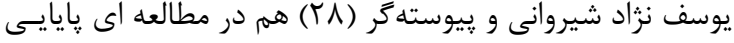

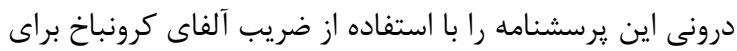

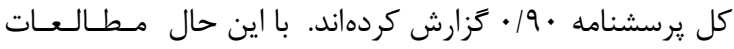

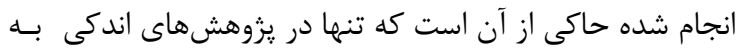
بررسى ويزّكى هاى روانسنجى

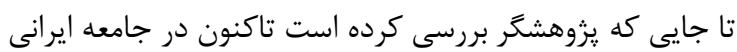

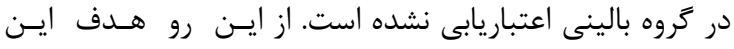

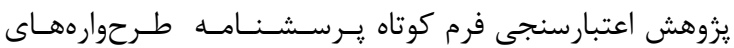

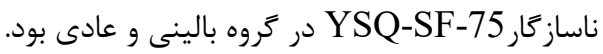
روش كار اين يزوهش از نوع مقطعى و اعتبارسنجى ابزار بود. براى تعييـن

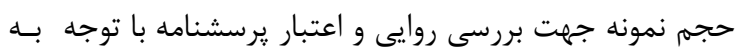

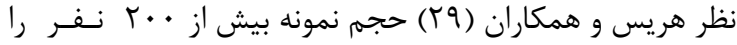

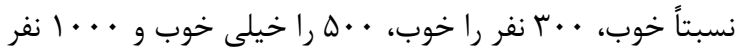

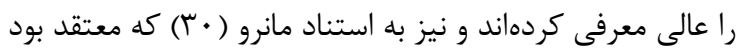

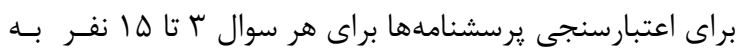

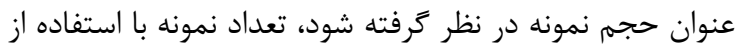

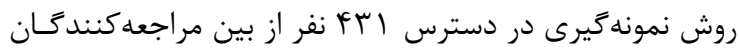

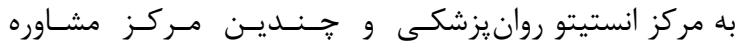

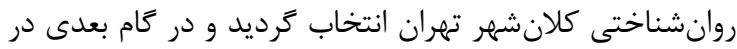

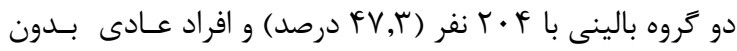

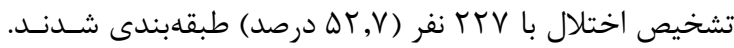

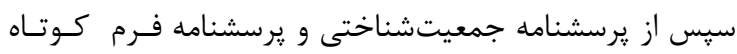

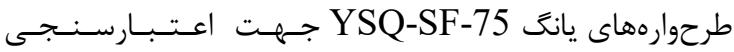

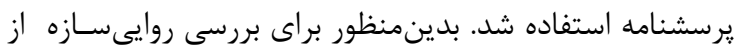

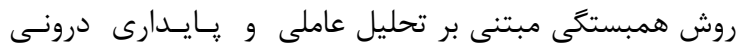

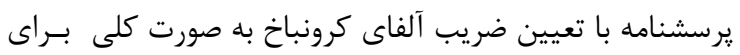

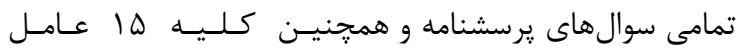

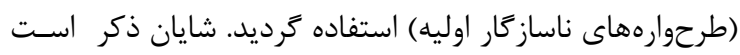

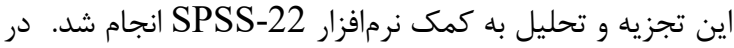

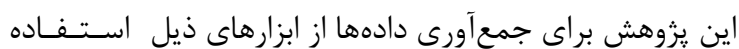
كرديد:

يرسشنامه جمعيتشناختى محققساخته: اين برسشنـامـهـ

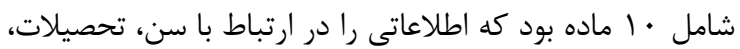

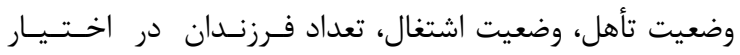

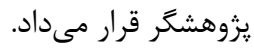

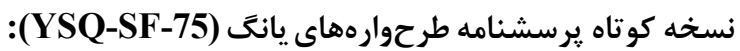

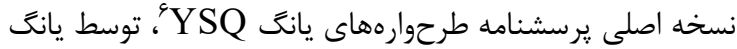

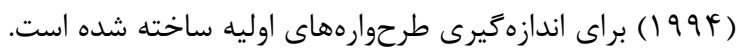

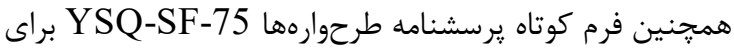

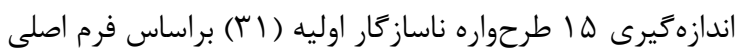


جدول ا: ويزَكى هاى جمعيت شناختى نمونه هاى بزوهش

\begin{tabular}{|c|c|c|c|}
\hline كروه عادى & كروه بالينى & & \\
\hline$(\% \Delta \Delta / q) \mid r V$ & (/ & آقا & \multirow{2}{*}{ جنسيت } \\
\hline$(\% F Y / V) 9 V$ & $(/ .99 / T) 1 T \Delta$ & خانم & \\
\hline$(\%, r \mid / q) \& q$ & سז (19/1 (\%) & دييلم و پايينتر & \multirow{4}{*}{ تحصيلات } \\
\hline$(1.91 / 7) 11 \%$ & $(/ . V / \varphi) / Q \cdot$ & كاردانى تا ارشد & \\
\hline (\% & $(\% \Lambda / \Lambda) \backslash \Lambda$ & دكترا & \\
\hline$(\% \mid r / T) \Gamma \cdot$ & $(\% 1) r$ & 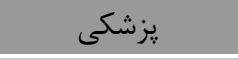 & \\
\hline$(\% \vee V \wedge / F) \backslash \Delta q$ & $(/ \Delta) / \Delta)) \cdot \Delta$ & مجرد & \multirow{3}{*}{ وضعيت تاهل } \\
\hline$(\% \cdot r \cdot \mid 9) Y^{F}$ & $(/ . F Y / Q) \wedge \vee$ & متاهل & \\
\hline$(\% 1) r$ & $(\% . \Delta / 9) \mid r$ & مطلقه & \\
\hline$(/ . r / \Delta) \vee$ & $(\% \mid r / r) r q$ & 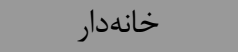 & \multirow{6}{*}{ وضعيت اشتغال } \\
\hline$(\% \vee F / q) \mid f q$ & (\% $\mid \wedge / 9) \Gamma \wedge$ & دانشجو & \\
\hline r & $(\% r \Delta / \Delta) \Delta r$ & كارمند & \\
\hline$(\% 1) r$ & $(/ . F / f) q$ & معلم & \\
\hline r & $(\% .19 / V) \mu F$ & شغل آزاد & \\
\hline$(/ \Gamma / \Delta) \Delta$ & rr (1 & ساير & \\
\hline$(\% \vee \wedge / V) \mid f$. & $(/ . \vee q) 1 \ldots$ & زندگى مشترك & \multirow{3}{*}{ ارتباط والدين با هم } \\
\hline$(/ . \Delta / 9) 1 \cdot$ & سז (T/ & طلاق & \\
\hline$(\%, Q / V) r \Lambda$ & $(\%$ TY/D) FQ & اختلاف بين والدين & \\
\hline
\end{tabular}

مقايسهاى، شاخص برازندگى غيرهنجار بنتلر - بونت NNFI' '.

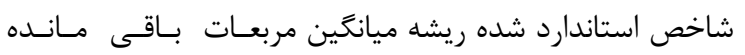

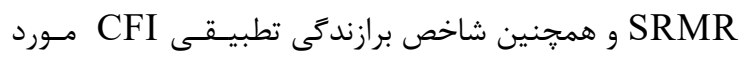

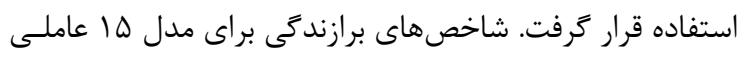
¿2/df=2/80 ،df=2595 $\chi 2=7280 / 2$ ) ،NNFI $=0 / 97 \quad$ CCFI $=0 / 97 \quad$,GFI $=0 / 69$

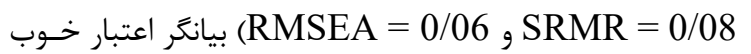

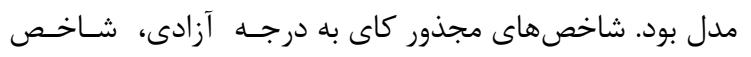

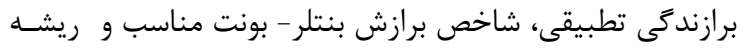

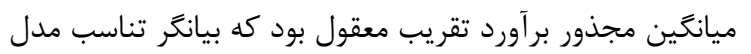

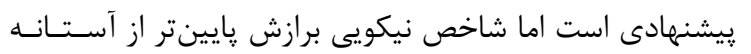

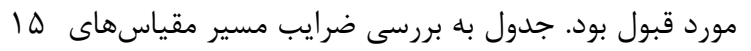

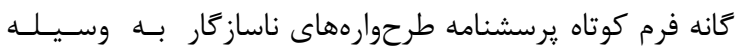
آيتمهاى يرسشنامه خواهد يرداخت.

10- Non-Normed Fit Indexes
براى برآورد يارامترهاى مدل از روش بيشينه احتمال استــــاده

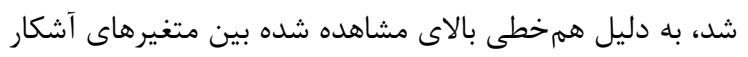

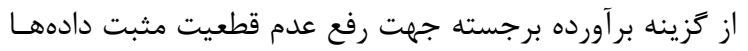

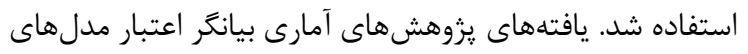

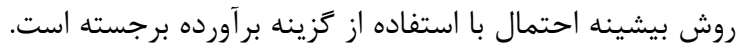

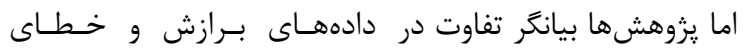

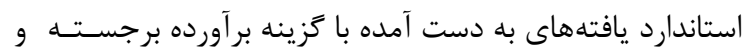

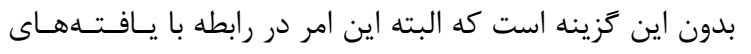

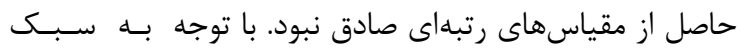

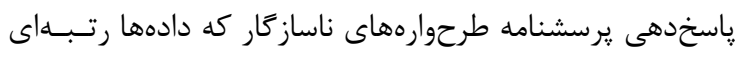

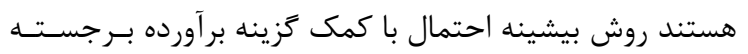
براى يزوهش حاضر بلامانع است.

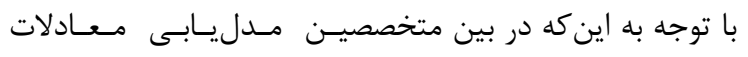

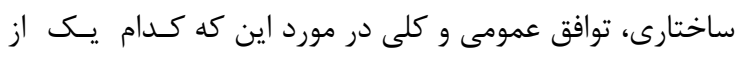

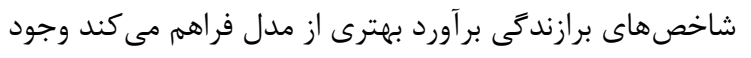

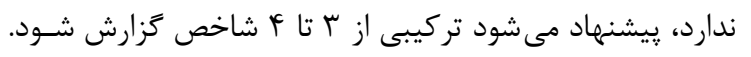

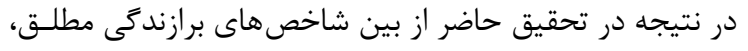

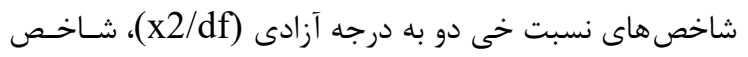

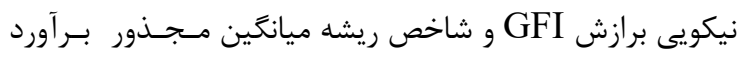

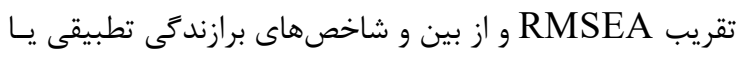


جدول ז: ضرايب مسير مقياسهاى ها كانه فرم كوتاه يرسشنامه طرحوارههاى ناساز

\begin{tabular}{|c|c|c|c|c|c|c|c|c|}
\hline ضريب مسير & مقياس & آيتم & ضريب مسير & مقياس & آيتم & ضريب مسير & مقياس & آيتم \\
\hline$\cdot / 4 \Delta$ & SS & $\Delta 1$ & $\cdot / V V$ & FA & rq & $\cdot \mid \Lambda \Delta$ & ED & .1 \\
\hline$\cdot 1 \Lambda$ & SS & $\Delta T$ & - /ve & FA & TV & . 119 & ED & r. \\
\hline$\cdot / V V$ & SS & $\Delta r$ & $\cdot|\lambda|$ & FA & $r \Lambda$ & $\cdot / V V$ & ED & r. \\
\hline . /VE & SS & $\Delta F$ & . /AV & FA & rq & - IAT & ED & f \\
\hline .199 & SS & $\Delta \Delta$ & $\cdot|\wedge|$ & FA & $r$. & .191 & ED & $\Delta$ \\
\hline$\cdot / 1 / 9$ & EI & $\Delta \varphi$ & $\cdot / \Lambda$ & DI & r & - IAT & $\mathrm{AB}$ & .9 \\
\hline$\cdot / \wedge \Delta$ & EI & $\Delta V$ & $\cdot / \Lambda$ & DI & rT & - IAT & $\mathrm{AB}$ &.$V$ \\
\hline - IAT & EI & $\Delta \Lambda$ & . /VG & DI & r & $\cdot 1 \wedge \Delta$ & $\mathrm{AB}$ & .1 \\
\hline$\cdot / V T$ & EI & $\Delta q$ & . IV & DI & re & . 194 & $\mathrm{AB}$ & .9 \\
\hline .149 & EI & 9. & - IAS & DI & ra & $.19 \mathrm{~V}$ & $\mathrm{AB}$ & .1. \\
\hline - IVF & US & 91 & $\cdot|\Lambda|$ & $\mathrm{VH}$ & rq & $\cdot / V T$ & MA & .11 \\
\hline . IVG & US & Gर & $\cdot / \wedge \Delta$ & VH & rV & . IVT & MA & $.1 T$ \\
\hline . 194 & US & qr & - IAT & $\mathrm{VH}$ & $r \Lambda$ & $\cdot 1 \wedge \Delta$ & MA & r \\
\hline$\cdot|V|$ & US & glf & $\cdot 1 \mathrm{~A}$ & VH & rq & $\cdot|\Lambda|$ & MA & $.1 f^{\mathrm{f}}$ \\
\hline$\cdot \mid \Delta \Lambda$ & US & 90 & $\cdot|V|$ & VH & f. & .109 & MA & .10 \\
\hline .194 & ET & 99 & $\cdot / V V$ & EM & if & $\cdot / V Q$ & SI & .19 \\
\hline$\cdot / V \Lambda$ & ET & SV & $\cdot|V|$ & EM & Fr & $\cdot 10$ & SI & $.1 \mathrm{~V}$ \\
\hline .190 & ET & 91 & $\cdot / V$ & EM & Fr & $\cdot / V 9$ & SI & .11 \\
\hline . & ET & 99 & . /V 9 & EM & Fq & $\cdot \mid \Lambda \Lambda$ & SI & .19 \\
\hline$\cdot|V|$ & ET & v. & $\cdot / V V$ & EM & EQ & $\cdot \mid \mathrm{A} \Lambda$ & SI &.$r$. \\
\hline .19 & IS & VI & $\cdot|V|$ & SB & is & $\cdot / V V$ & DS &.$r I$ \\
\hline$\cdot / V \wedge$ & IS & VT & - $/ A r$ & SB & FV & . IAT & DS & . T \\
\hline$\cdot|0|$ & IS & $V r$ & •/A & SB & $\forall \wedge$ & - $/ \lambda F$ & DS & זr. \\
\hline$\cdot 1 \Delta \Delta$ & IS & $V F$ & $\cdot \mid \wedge 9$ & SB & 19 & $\cdot \mid A V$ & DS & TF \\
\hline$\cdot / V T$ & IS & $v \Delta$ & $\cdot / v \wedge$ & SB & $\Delta$. & $\cdot / A F$ & DS & $r \Delta$ \\
\hline
\end{tabular}

در ميان مقياسهاى فرم كوتاه يرسشنامه طرحوارههاى ناسازكار

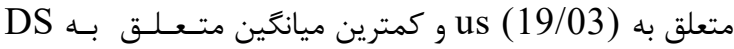

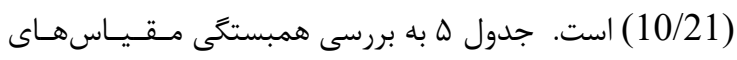
استخراج شده مى يردازد.

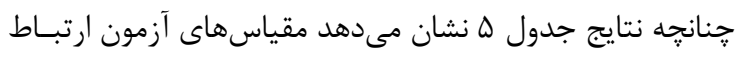

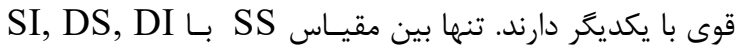

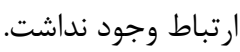

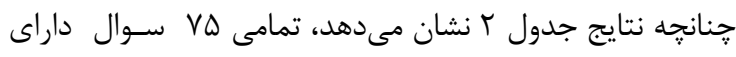

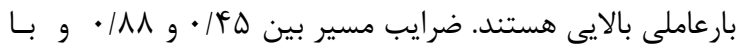

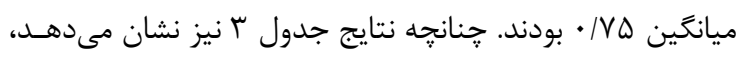

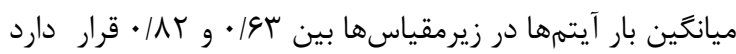

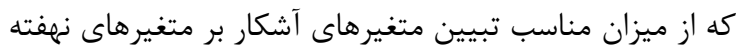

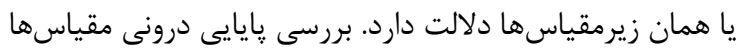

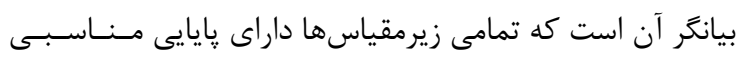

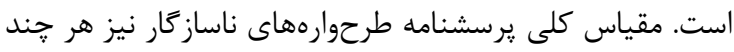

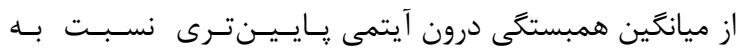

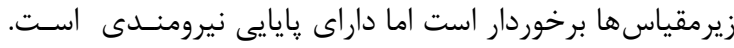

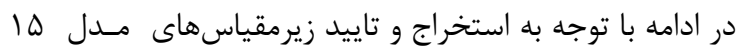

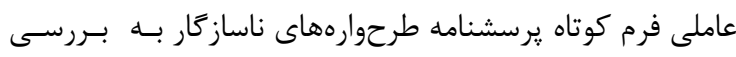

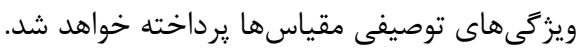

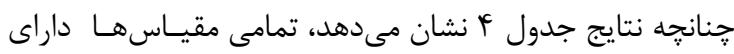

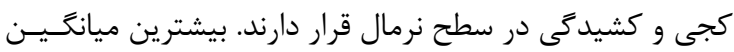




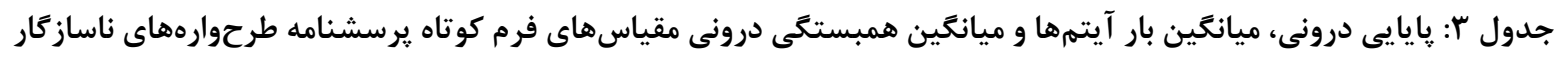

\begin{tabular}{|c|c|c|c|c|}
\hline آلفاى كرونباخ استاندارد شده & ميانكين همبستَى درون آيتمى & ميانكين بار آيتمها & تعداد آيتم & مقياسها \\
\hline$\cdot / 19$ &. $\mid 94$ & $\cdot / 199$ & $\Delta$ & ED \\
\hline$\cdot / 19$ & $\cdot 109$ & - / V $\Delta$ & $\Delta$ & $\mathrm{AB}$ \\
\hline$\cdot / 1 \Delta \Delta$ &.$/ Q F$ & $\cdot / \mathrm{N}$ & $\Delta$ & MA \\
\hline$\cdot / 1 \Delta \Delta$ & . $/ \Delta T$ & . IVG & $\Delta$ & SI \\
\hline.$/ 9$ & .199 & . IAt & $\Delta$ & DS \\
\hline$\cdot 119$ & . & $\cdot / \Lambda$. & $\Delta$ & FA \\
\hline .119 & .194 & $\cdot / 1 / 9$ & $\Delta$ & DI \\
\hline$\cdot / 1 \Lambda$ & .19. & $\cdot / v 9$ & $\Delta$ & VH \\
\hline$\cdot / \wedge \Delta$ & . & $\cdot / V^{A}$ & $\Delta$ & EM \\
\hline .119 & .195 & $\cdot / \Lambda$ & $\Delta$ & SB \\
\hline . IAT & $\cdot / 4 \wedge$ & .191 & $\Delta$ & SS \\
\hline$\cdot / \Lambda F$ & $\cdot / \Delta T$ & $\cdot / V T$ & $\Delta$ & EI \\
\hline$\cdot / \Lambda$ & $\cdot / 4 \Delta$ & .191 & $\Delta$ & US \\
\hline$\cdot / \Lambda$ & $\cdot / 4 \Delta$ & .191 & $\Delta$ & ET \\
\hline . IV & $\cdot \pi 9$ & $.19 \pi$ & $\Delta$ & IS \\
\hline .199 & $\cdot / r \Lambda$ & $\cdot / V F$ & $V \Delta$ & كل \\
\hline
\end{tabular}

جدول fا: ويزَى هاى توصيفى مقياسهاى فرم كوتاه يرسشنامه طرحوارههاى ناسازكار

\begin{tabular}{|c|c|c|c|c|c|c|}
\hline كشيدكى & كجى ل كى & انحراف استاندارد & ميانكَين & بيشينه & كمينه & مقياسها \\
\hline-.94 & . & $\mathrm{V} / \mathrm{I}$ & $1 \% / V G$ & $r$. & $\Delta$ & ED \\
\hline$-\cdot / \Lambda \gamma$ & $\cdot / \pi \Delta$ & GIAV & $10 / V 9$ & $r$. & $\Delta$ & $\mathrm{AB}$ \\
\hline$-\cdot / r V$ & $\cdot|V|$ & $9 / 1 \pi$ & $14 / 11$ & r. & $\Delta$ & MA \\
\hline •199 & $1 / 11$ & $\Delta / 9 V$ & $11 / r \Lambda$ & $r$. & $\Delta$ & SI \\
\hline $1 / 1 \mathrm{r}$ & $1 / \pi \Lambda$ & $9 / 10$ & $1 \cdot / r 1$ & $r$. & $\Delta$ & DS \\
\hline$\cdot / 4 \Lambda$ & $1 / 1 r$ & $9 / T^{2}$ & $11 / T V$ & r. & $\Delta$ & FA \\
\hline $1 / \cdot r$ & $1 / r T$ & 91.4 & $1 \cdot / \& \Delta$ & $r$. & $\Delta$ & DI \\
\hline.$/ \cdot \mathrm{f}^{2}$ &.$/ 9 V$ & $9 / 4 \wedge$ & $11 / 79$ & $r$. & $\Delta$ & VH \\
\hline .110 & .199 & $9 \pi \Delta$ & $11 / 94$ & r. & $\Delta$ & EM \\
\hline-.1 .9 & .19 & 9109 & $11 / 99$ & r. & $\Delta$ & SB \\
\hline$-\cdot / \Delta \Delta$ & $-\cdot / 1 \Lambda$ & $\Delta / V^{F}$ & IV/94 & r. & $\Delta$ & SS \\
\hline$-\cdot / 4 T$ & .190 & $9 / \pi \Lambda$ & $\mid r / T V$ & r. & $\Delta$ & EI \\
\hline.$- / r q$ &.$- / 1 \Lambda$ & $\Delta / 9 F$ & $19 / \cdot r$ & $r$. & $\Delta$ & US \\
\hline-.199 & $\cdot|r|$ & 91.4 & $19 / 49$ & $r$. & $\Delta$ & ET \\
\hline$-\cdot / 4 r$ & 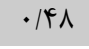 & $\Delta / \uparrow \wedge$ & $1 \% / \Delta$. & r. & $\Delta$ & IS \\
\hline
\end{tabular}


جدول ه: همبستخى مقياسهاى فرم كو تاه يرسشنامه طرحوارههاى ناسازكًار

\begin{tabular}{|c|c|c|c|c|c|c|c|c|c|c|c|c|c|c|}
\hline & ED & AB & MA & SI & DS & FA & DI & VH & EM & SB & SS & EI & US & ET \\
\hline AB & $.366^{* *}$ & & & & & & & & & & & & & \\
\hline MA & $.557^{* *}$ & $.475^{* *}$ & & & & & & & & & & & & \\
\hline SI & $.551^{* *} .325^{* *}$ & $.663^{* *}$ & & & & & & & & & & & \\
\hline DS & $.626^{* *} .492^{* *}$ & $.654^{* *}$ & $.669^{* *}$ & & & & & & & & & & \\
\hline FA & $.520^{* *}$ & $.514^{* *}$ & $.485^{* *}$ & $.513^{* *}$ & $.729^{* *}$ & & & & & & & & & \\
\hline DI & $.391^{* *}$ & $.558^{* *}$ & $.422^{* *}$ & $.450^{* *}$ & $.625^{* *}$ & $.756^{* *}$ & & & & & & & & \\
\hline VH & $.478^{* *} .536^{* *}$ & $.587^{* *}$ & $.510^{* *}$ & $.688^{* *}$ & $.628^{* *}$ & $.636^{* *}$ & & & & & & & \\
\hline EM & $.230^{* *} .532^{* *}$ & $.403^{* *}$ & $.373^{* *}$ & $.448^{* *}$ & $.457^{* *}$ & $.580^{* *}$ & $.512^{* *}$ & & & & & & \\
\hline SB & $.487^{* *} .554^{* *}$ & $.548^{* *}$ & $.488^{* *}$ & $.650^{* *}$ & $.692^{* *}$ & $.692^{* *}$ & $.640^{* *}$ & $.582^{* *}$ & & & & & \\
\hline SS & $.203^{* *} .223^{* *}$ & $.157^{* *}$ & .035 & .093 & $.140^{* *}$ & .059 & $.164^{* *}$ & $.151^{* *}$ & $.253^{* *}$ & & & & \\
\hline EI & $.475^{* *} .334^{* *}$ & $.490^{* *}$ & $.480^{* *}$ & $.550^{* *}$ & $.493^{* *}$ & $.411^{* *}$ & $.505^{* *}$ & $.339^{* *}$ & $.503^{* *}$ & $.256^{* *}$ & & & \\
\hline US & $.284^{* *}$ & $.297^{* *}$ & $.498^{* *}$ & $.303^{* *}$ & $.279^{* *}$ & $.171^{* *}$ & $.121^{*}$ & $.303^{* *}$ & $.200^{* *}$ & $.261^{* *}$ & $.445^{* *}$ & $.376^{* *}$ & & \\
\hline ET & $.268^{* *}$ & $.266^{* *}$ & $.471^{* *}$ & $.387^{* *}$ & $.370^{* *}$ & $.223^{* *}$ & $.227^{* *}$ & $.383^{* *}$ & $.247^{* *}$ & $.263^{* *}$ & $.197^{* *}$ & $.379^{* *}$ & $.512^{* *}$ & \\
\hline IS & $.355^{* *} .416^{* *}$ & $.436^{* *}$ & $.410^{* *}$ & $.458^{* *}$ & $.536^{* *}$ & $.526^{* *}$ & $.476^{* *}$ & $.366^{* *}$ & $.516^{* *}$ & $.135^{* *}$ & $.459^{* *}$ & $.262^{* *}$ & $.440^{* *}$ \\
\hline
\end{tabular}

**.Correlation is significant at the 0.01 level (2-tailed).

*.Correlation is significant at the 0.05 level (2-tailed).

غيربالينى با استفاده از ضريب آلفاى كرونباخ در جمعيت مونـث

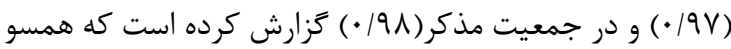

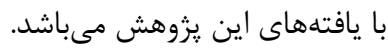

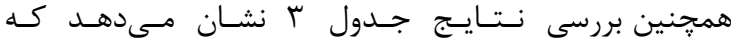

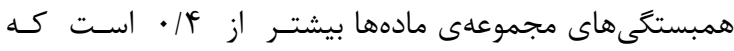

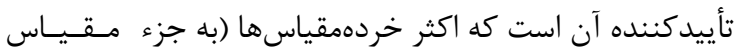

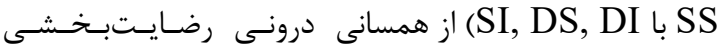

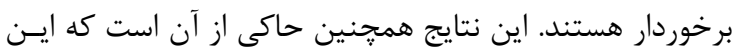

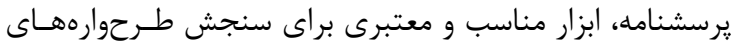

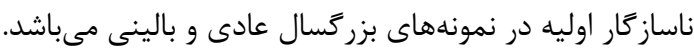

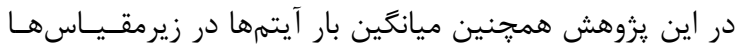

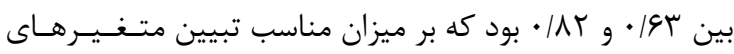

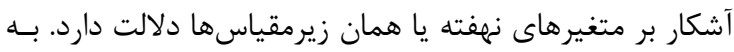

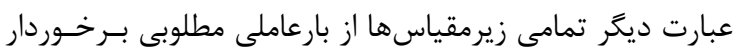

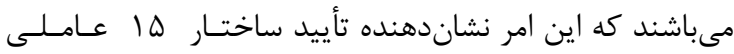

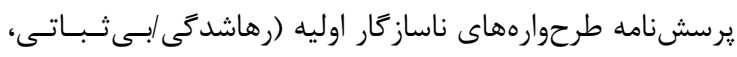

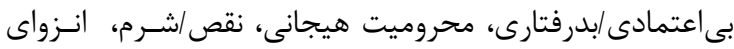

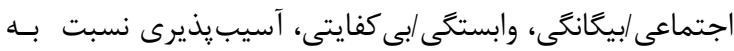

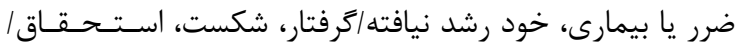

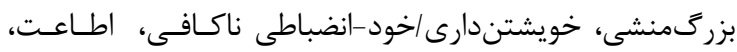

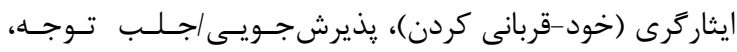

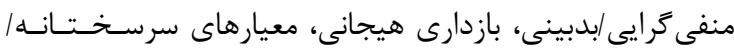

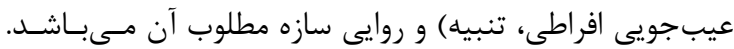

بحث و نتيجه

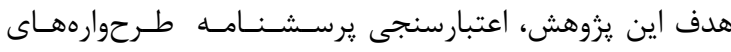

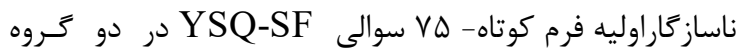

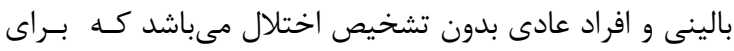

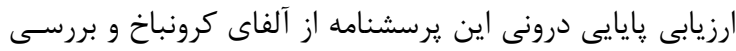

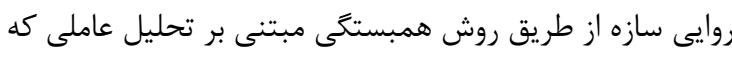

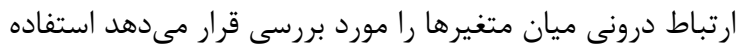

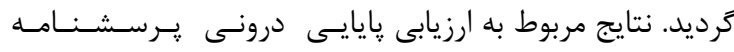
طرحوارههاى ناساز

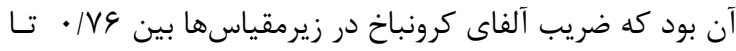

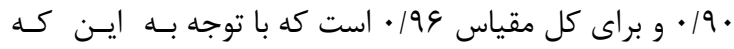

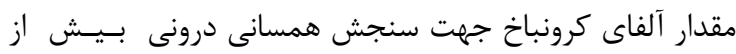

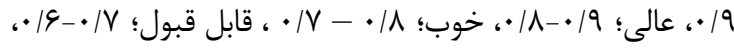

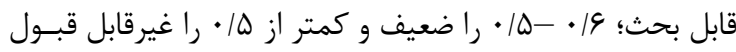

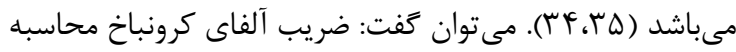

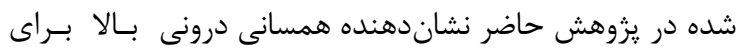

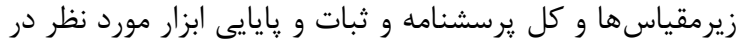

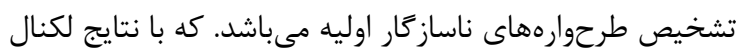

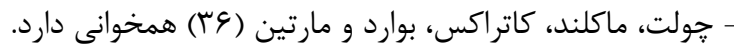

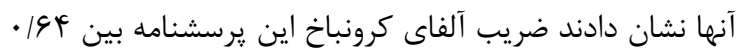

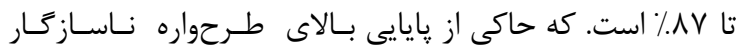

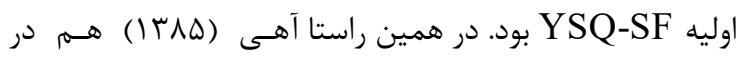

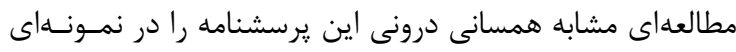




\section{References}

1- Nasiri M, Zarea K, Nasiri M, Saidkhani V. Therelationship between attitude toward Islamic coveringand mental health among the students of BushehrUniversity of Medical Sciences. Jentashapir Jof Health Res. 2014; 6(3): 107-115.

2- Ghahari Sh, Davoodi R, Yekehfallah M, Mazloumi Rad, M. Marital Conflict, Cognitive Emotion Regulation, Maladaptive Schema and Sexual Satisfaction in spouse abused and nonabused women in Iran: A comparative study, 2018. Asian journal of Psychiatry. 2018;(35):1-2. 3- Moradi M, Faqihi A.N. The relationship between parenting and early dysfunctional schemas. Islamic Research Journal of Women and Family. 2018; 5(8): 23-48.

4- Zhang D.H. He H.L. Personality traits and life satisfaction: A Chinese case study. Social Behavior and Personality.2010; 38(8): 1119-1122. 5- Csukly G, Telek R, Filipovits D, Takács B, Unoka Z, Simon L. What is the relationship between the recognition of emotions and core beliefs:associations between the recognition of emotions in facial expressions and the maladaptive schemas in depressed patients? J Behav Ther Exp Psychiatry 2010. 2011;42(1):129-37. 6- Dehghani S, Izadikhah Z, Mohammad Taghi Nasab M, Rezaei A. Dysfunctional schema, coping strategies and quality of life. Journal of Behavioural Research. 2015; 12 (9): 1-8.

7- Young JE, Klosko JS, Weishaar ME. Schema Therapy: A Practitioners Guide. Translated by Hamidopur \& Andooz. Tehran: Arjmand. 2007;29-92.

8- Roelofs J, Onckels L, Muris P. Attachment Quality and Psychopathological Symptoms in Clinically Referred Adolescents: The Mediating Role of Early Maladaptive Schema. Journal of Child and Family Studies. 2013; 3(22): 377-85.

9- Sobhi Gharamelki N, Parzour P, Aghajani S, Narimani M. The efficacy of emotion regulation skills on distress. Anxiety and depression in university students. Journal of Health education and health Promotion. 2016; 1 (1): 5-13.

10- Young JE, Klosko JS, Weishaar ME. [Schema therapy: A practitioner's guide]. Hamidpour H, Andooz Z. (translators). Tehran: Arjmand; 2003: 23-41.

11- Zeigler-Hill V, Green B.A, Arnau R.C, Sisemore T.B. Myers E.M. Trouble ahead, trouble behind: Narcissism and early maladaptive schemas. Journal of Behavior Therapy and Experimental Psychiatry.2011; 42(1): 96-103.

12- Agha Yousefi A, Amirpour b. Psychometric propertice of children schema questionnair journal of shahid Beheshti university of Medical Sciences. 2012; 5 (89): 265-271.

13- Batool N, Shehzadi H., Riaz M.N. Riaz M.A. paternal malparenting and offspring personality disorders: Mediating effect of early
شاخصهاى برازش مدل مربوطه هم نشان دهنده برازش عـالى إنى

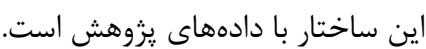

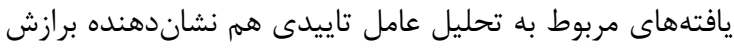

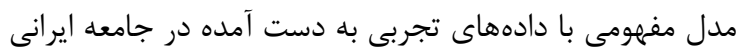

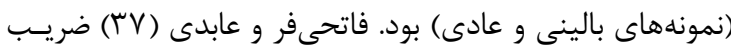

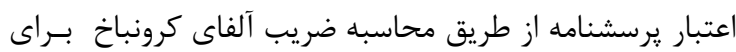

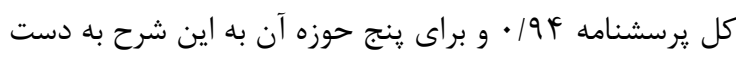

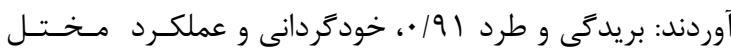

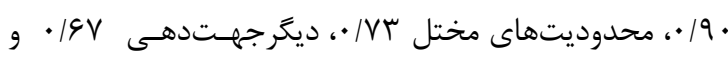

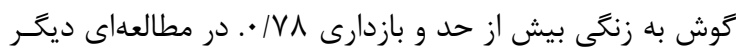

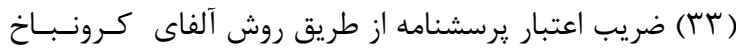

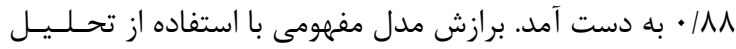

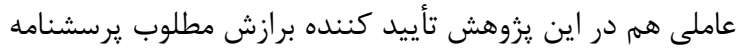

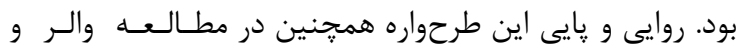

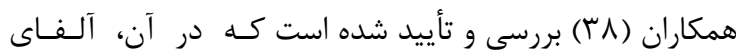

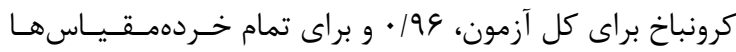

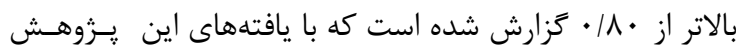
همسو مىباشد.

بنابراين زرسشنامه حاضر به دليل برخوردارى از روايى و اعتبـار

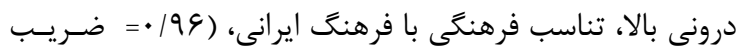

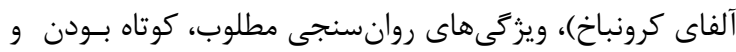

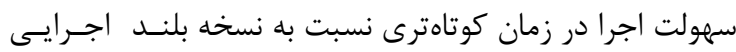

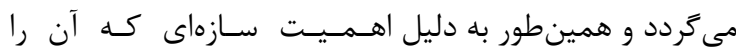

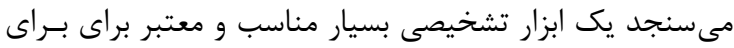

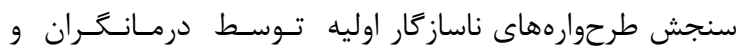

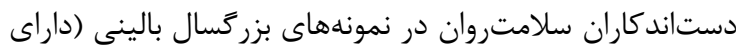

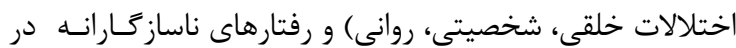

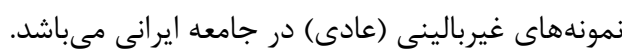
تقدير و تشكر

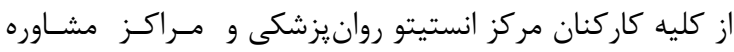

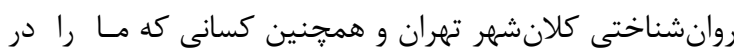
انجام اين يزوهش يارى رساندند، تقدير و تشكر مىنمائيمه ونمئ 
maladaptive schemas. Journal of the Pakistan Medical Association.2017; 67(4): 556-560.

14- Oei T.P, Baranoff J. Young schema questionnaire: review of psychometric and measurement issues. Australian Journal of Psychology.2007; 59: 78-86.

15- Reeves M, Taylor J. Specific relationships between core beliefs and personality disorder symptoms in a non-clinical sample. Clinical Psychology and Psychotherapy. 2007; 14: 96-104.

16- Batmaz S, Kaymak S.U, Kocbiyik S. Turkcapar M.H. Metacognitions and emotional schemas: a new cognitive perspective for the distinction between unipolar and bipolar depression. Comprehensive Psychiatry.2014; 55(7): 1546-55 17- Sava F.A. (2009). Maladaptive schemas, irrational beliefs, and their relationship with the five-factor personality model. Journal of Cognitive and Behavioral Psychotherapies. 2009; 9: 135-141.

18- Bahramizadeh H, Ehsan H.B. Early Maladaptive Schemas and Agreeableness in Personality Five Factor Model. Procedia - Social and Behavioral Sciences. 2011; 30: 547 - 551.

19- Flink N, Lehto S.M, KoivumaaHonkanen H, Viinamäki H, Ruusunen A, Valkonen M. Korhonen H.K. Early maladaptive schemas and suicidal ideation in depressed patients. The European Journal of Psychiatry.2017; 31(3): 92-87.

20- Calvete, E. Emotional abuse as a predictor of early maladaptive schemas in adolescents: Contributions to the development of depressive and social anxiety symptoms. Child Abuse \& Neglect. 2014; 38 (4):735-746.

21- Orue I, Calvete E. Padilla P. Brooding rumination as a mediator in the relation between early maladaptive schemas and symptoms of depression and social anxiety in adolescents. Journal of Adolescent Health. 2014; 3(8): 91111.

22- Young J.E. Young Schema Questionnaire Short Form. New York: Cognitive Therapy Center. 1999.

23- Ahi GH, Psychometric properties of schemas questionnaire-short from. Master thesis of shahid Beheshti universitgeneral psychology. Allameh Tabatabaee University.2007.

24- Hamidpour H, Andouz Z. [Translation of Schema therapy]. Young JE, Klosko JS, Weishaar ME (Authors). Tehran: Arjmand Publications; 2014. [Persian].

25- Welburn K, Coristine M, Dagg P, Pontrefact A, Jordan S. The Schema QuestionnaireShort Form: Factor analysis and relationship between schemas and symptoms. Cognitive Therapy and Research.2002; 26: 519530.

26- Baranoff J, Oei T.P.S, Cho S.H, Kwon S. $\mathrm{M}$. Factor structure and internal consistency of the Young Schema Questionnaire (Short Form) in Korean and Australian samples. Journal of Affective Disorders.2006; 93: 133-140.

27- Wellburn K, Dagg P, Coristine M. Pontrefact, A. Schematic change as a result of an intensive group-therapy day-treatment program. Psychotherapy: Theory, Research, Practice, Training. 2000; 37: 189-195.

28- Yoosofnejad Shirvani M, Peyvastegar M. The relationship between life satisfaction and early maladaptive schemas in university students. Knowledge \& Research in Applied Psychology. 2011; 2(44):55-65.

29- Harris A, Curtin L. Parental perceptions, early maladaptive schemas, and depressive symptoms in young adults. Cognit Ther Res 2002; 26: 405-16.

30- Munro BH, Statistical methods for health care research: Lippincott Williams and Wilkins; 2005.

31- Yousefi R. The Comparison of Early Maladaptive Schemas and Cognitive Emotion Regulation Styles in Patient with Functional Gastrointestinal Disorders and Normal Group. Govaresh 2015; 19:257-64.

32- Nordahl. Hans M, Holth, Haugum. Ion A. Early Maladaptive schemas in Patient with or without personality disorders clinical psychology and psychotherapy. 2005; 12: 142-149.

33- Yousefi R. The study effectiveness and comparison of schemafocused and life stylefocused group training intervention in marital satisfaction enhancement. Doctoral Dissertation.Tehran; Shahid Beheshti University 2010 [Persian].

34- Sijtsma, K. On the use, the misuse and the very limited usefulness of Cronbach's alpha, Psychometrika. 2009; 74: 107-120.

35- Mohammadbeigi A, Mohammadsalehi N, Aligol M. Validity and Reliability of the Instruments and Types of MeasurmentS in Health Applied Researches, Journal of Rafsanjan University of Medical Sciences.2015; 13: 1153-1170.

36- Hamidpour H, Dolatshahi B, Porshahbaz A, Dadkhah A. The efficacy in women with GAD. Journal of Psychiatry and Clinical Psychology. 2010; 4: 420-431.

37- Sadooghi Z, Vafaiee M, Rasoolzadeh K, Esfahanian N. Factor analysis short-form Young schema questionnaire in Iranian nonclinical sample. Iran J Psychiatry Clinical Psychology.2007; 4: 209-214.[Persian].

38- Waller G, Meyer C, Ohanian V. Psychometric properties of the long and short versions of the Young Schema Questionnaire. Core beliefs among bulimic and women. Cogn Ther 2001; 25(2): 137-47. 